\title{
Therapeutic targets in osteoarthritis: from today to tomorrow with new imaging technology
}

\author{
J-P Pelletier, J Martel-Pelletier
}

Ann Rheum Dis 2003;62(Suppl II):ii79-ii82

O ver the last decade, there have been several interesting advances in the treatment of osteoarthritis (OA). A clearer understanding of the pathophysiology of this disease $^{1}$ has facilitated the development of new approaches for treatments aimed at specifically and effectively retarding disease progress. New classes of molecules that inhibit one or more OA catabolic processes are under evaluation for their potential to alter the degenerative process.

OA can be described as the degradation and loss of articular cartilage accompanied by subchondral bone remodelling, osteophyte formation, and synovial membrane inflammation. This is clinically reflected by gradual development of fluctuating joint pain, swelling, stiffness, and loss of motion. Pharmacological interventions have focused on treating pain, primarily using non-steroidal anti-inflammatory drugs (NSAIDs), analgesics, and, more recently, specific cyclooxygenase-2 (COX-2) inhibitors. The rationale behind this was to inhibit COX, the key enzymes that metabolise arachidonic acid to prostaglandins.

\section{THERAPEUTICS IN OSTEOARTHRITIS}

Clinical studies to date have focused on the alleviation of signs and symptoms, for which comparable efficacy has generally been found for NSAIDs and acetaminophen in stable cohorts of patients with mild to moderate OA. ${ }^{2}{ }^{3}$ Published data on the use of intra-articular corticosteroids for OA have shown short term (up to four weeks) improvement in signs and symptoms compared with placebo. ${ }^{24}$ The comparative efficacy of these agents in the treatment of episodic crystal induced inflammatory exacerbations superimposed on chronic OA in selected patients, ${ }^{5}$ for which one might predict the superior efficacy of NSAIDs or corticosteroids over acetaminophen, has not been studied. However, the main objectives in the management of $\mathrm{OA}$ are not only to reduce symptoms and minimise functional disability, but also to limit progression of structural changes.

As there are in vitro data to indicate that corticosteroids inhibit synoviocytes and chondrocyte production of interleukin 1 (ILl), COX-2, and tumour necrosis factor $\alpha,{ }^{4}$ there has been speculation that intra-articular corticosteroid administration may have a disease modifying effect in OA. However, a recent structural outcome study in humans did not validate such a hypothesis. ${ }^{6}$ Similarly, despite speculation that NSAIDs may exert beneficial or harmful effects on the integrity of articular cartilage, there are no validated imaging studies that shed light on this controversy. Predicting the net effects of COX inhibitors on cartilage structure is particularly difficult given the observed pleiotropic effects of individual eicosanoids in vitro on chondrocyte functions as discussed above. Thus, although the long term effects of available anti-inflammatory agents on cartilage merit further investigation, there is significant interest in new agents that have the potential to reduce or stop the progression of structural changes observed in OA. Such agents offer great promise and are likely to lead to very significant changes in therapeutic approaches in the near future.

\section{INFLAMMATION IN OSTEOARTHRITIS}

The most attractive recent findings are the data pointing to an association between inflammation and disease appearance and progression. ${ }^{7}$ There are several studies in which the levels of biomarkers such as cartilage oligoprotein, hyaluronic acid, or $\mathrm{C}$ reactive protein were measured in patients with OA. These studies showed that there is a strong correlation between synovial inflammation and OA progression as well as an association with strong risk factors. It is generally believed that clinical signs of an active disease suggest the likelihood of rapid progression of the disease. Moreover, there are a number of pathways linked to inflammation that represent the most interesting therapeutic targets. For instance, cytokines, such as ILI $\beta$ and other proinflammatory cytokines or inflammatory factors, are probably responsible for the signs and symptoms of inflammation present in patients with OA. There are a number of ways by which the production or activity of cytokines could be reduced. The action of cytokines can be reduced at the cell membrane level by decreasing the membrane receptor level or by the use of receptor antagonists or soluble receptors. The action of the cytokine can also be reduced by blocking the intracellular signalling pathways.

\section{CONTROLLING THE OSTEOARTHRITIC PROCESS}

We have shown that the natural ILl receptor antagonist (ILIRa) is capable of reducing several cartilage catabolic processes, which are ILI $\beta$ dependent. Indeed, in vivo studies in animal models have shown that the intra-articular injection of ILlRa could block the action of ILl $\beta$ and reduce disease progression. ${ }^{8}$ Studies using gene therapy and different gene transfection methods have allowed the successful in vivo transfection of the ILlRa gene into the OA knee joints of dogs and rabbits. ${ }^{9}{ }^{10}$ These studies have also shown that such therapeutic intervention could reduce the progression of OA lesions.

Another interesting target for controlling the activity of the ILl system is the ILl converting enzyme (ICE)/caspase l, which is responsible for the conversion of the proform of ILl $\beta$ into its active (mature) form. ${ }^{11}$ A number of studies are underway on the use of an orally active ICE inhibitor in patients with rheumatoid arthritis. This form of treatment could also be used for OA.

The role of proteases in the degradation of the extracellular matrix of cartilage in OA has been well documented, and matrix metalloproteinases (MMPs) are believed to be important. Inhibition of the synthesis/activity of these enzymes as a treatment for OA has been the focus of intensive research. ${ }^{12}$ To date, the most promising strategy is the use of chemical molecules that can block the activity of MMPs. Certain MMPs,

Abbreviations: OA, osteoarthritis; COX, cyclo-oxygenase; IL, interleukin; ILIRa, IL I receptor antagonist; MMP, matrix metalloproteinase; MRI, magnetic resonance imaging 
such as MMP-13, have been selected as being the most attractive targets for the treatment of OA. Selective inhibition was chosen instead of broad inhibition to avoid side effects that may be related to a broad MMP inhibition. MMP action can be controlled in a number of ways, mainly by inhibiting their synthesis or the transformation of the proMMPs into active MMPs. The enzyme activity per se can be inhibited in many ways, including through the use of chemical inhibitors. Generally, these chemical inhibitors are chelators of heavy metals such as zinc and calcium. A number of these compounds that have a broad range of MMP activities have already been tested in clinical trials. These trials have shown that MMP inhibitors can produce side effects, and no major reduction in the progression of the disease has yet been found.

Another possible way to counter the effect of certain catabolic factors lies in the inhibition of certain intracellular signalling pathways such as the protein kinase cascades. They are responsible for transmitting the signal induced by the binding of the ligand to its receptor from the cell membrane to the nucleus, where it induces the cell response. Inhibitors of kinases, which are the most appealing targets, include p38, ERK 1, 2, and SAPK/JNK. A number of drugs designed to target these kinases are under development. In a recent study, ${ }^{13}$ we showed that orally active selective inhibitors of ERK 1, 2 could very significantly and effectively reduce the progression of lesions in the rabbit OA model, and also reduce the synthesis of catabolic factors that are ERK 1, 2 dependent, such as MMP. Another emerging field of research focuses on the development of molecules that can inhibit the binding of transcription factors at the DNA level. The major transcription factor targets for cytokines and MMPs are NF- $\mathrm{KB}$ and APl.

\section{NITRIC OXIDE}

Of the catabolic pathways activated by inflammation, synthesis of nitric oxide (NO) is an interesting one in the context of $\mathrm{OA}$ for at least two main reasons. Firstly, NO and its byproducts are capable of inducing the inflammatory component of OA. Secondly, they are also capable of inducing tissue damage and tissue destruction and therefore may be responsible not only for symptoms but also the disease process per se. It has been shown that the excess production of NO generated as the result of an increase in inducible NO synthase in OA tissues is injurious. This appears to occur through a number of mechanisms. To begin with, NO may induce chondrocyte death by apoptosis. It may also stimulate the synthesis and activities of MMP, and reduce the anabolism of cartilage by inhibiting the synthesis of the major matrix components, collagen and proteoglycan. Moreover, NO is capable of increasing the activity of COX-2 and consequently appears to be responsible for an increase in the signs and symptoms of the disease. Therefore it is believed that this molecule is a most interesting target, because reducing its excess production may not only reduce the symptoms but also reduce the progression of the disease, thereby reaching two targets simultaneously. This hypothesis is supported by studies using the experimental dog model of $\mathrm{OA}$ and the oral administration of therapeutic doses of a specific inhibitor of inducible NO synthase. ${ }^{14} 15$

\section{SUBCHONDRAL BONE}

Structural changes in OA also involve modifications of the morphology of the surrounding bone. Subchondral bone remodelling is a well recognised manifestation of OA. New data underline the concept that abnormal subchondral bone cell functions may contribute to the onset/progression of OA. Recent work suggests that, very early in the OA process, biological and morphological disturbances occur at the subchondral bone and may have a role in the modulation of articular cartilage metabolism. Therapeutic effects of drugs that prevent the abnormal metabolism of subchondral osteoblasts on the progression of OA lesions are of major interest for future intervention.

\section{METHODS FOR ASSESSING OSTEOARTHRITIS PROGRESSION}

Although several pharmacological agents that may be able to retard or inhibit the progression of structural changes in joint tissue are under investigation for the treatment of OA, the tools for studying such effects in the human population remain unsatisfactory. Sensitive and accurate methods for assessing the progression of $\mathrm{OA}$ are key requirements in research efforts to find new ways of controlling the disease.

The optimum method for measuring cartilage thickness remains radiography. Measurement of the joint space width (JSW) by standard radiography does not yield any information on the cartilage itself and gives only one measurement point; this practice considerably limits the statistical and clinical power of the technique in assessing cartilage degradation over time. Moreover, JSW, being a single point evaluation of bone to bone distance, produces only an approximate measurement of the overall thickness of the articular cartilage and gives no indication of its volume or any other structural changes associated with the disease process.

A recently described technique, arthroscopy (chondroscopy), allows assessment of the cartilage by direct visualisation with the use of an arthroscope. Although this technique appears to be reliable and sensitive to morphological change at one year, only the cartilage surface can be evaluated. Moreover, because this method is semiquantitative and invasive, large multicentre studies would be difficult to conduct.

Magnetic resonance imaging (MRI) allows precise visualisation of joint structures such as cartilage, bone, synovium, ligaments, and meniscus, and their pathological changes. ${ }^{16} 17$ Recent advances in this technology have led to significant improvement in spatial resolution and contrast, enabling researchers to evaluate anatomical damage of all these joint structures across sagittal, coronal, and axial planes. ${ }^{18-22}$ Although anatomical changes in the cartilage can be seen, quantification of these changes remains difficult. Initially, quantitative measurement of cartilage was studied in healthy subjects $^{232425}$ or animal models. ${ }^{26}$ Early work on cartilage in patients with $\mathrm{OA}$ was restricted to semiquantitative methods, ${ }^{27}{ }^{28}$ with some measurements in these patients correlating well with arthroscopy findings ${ }^{29}{ }^{30}$ and histological specimens. ${ }^{31}{ }^{32}$ Recently, the quantitative assessment of knee cartilage volume has become possible. ${ }^{33-42}$ There are few in vivo methods that allow sensitive, specific, valid, and reliable quantification of progression of $\mathrm{OA}$ of the knee. We have developed a method for the reliable evaluation of cartilage volume of the total knee (femur and tibia) using MRI sets acquired with fat suppressed, gradient echo sequences. ${ }^{43}$ Firstly, we evaluated the reliability of the novel imaging software system in controls and patients with OA. ${ }^{44}$ Secondly, we assessed the changes in cartilage volume in patients with knee OA that had MRI acquisition at baseline and a two year follow up. ${ }^{45}$ Finally, we determined whether other structural changes such as meniscal alteration could be associated as a risk factor for cartilage volume loss over time. ${ }^{46}$

For the first part, ${ }^{44} 48$ MRI examinations of the knee from normal subjects, from patients with different stages of symptomatic knee OA, and from a subset of duplicate images were independently and blindly quantified by three readers using our imaging system. The following cartilage areas were analysed to compute volumes: global cartilage, medial and lateral compartments, and medial and lateral femoral condyles. The results of this study established the reliability of this MRI system. Indeed, test-retest reliability, between reader agreement, and patient positioning reliability were excellent.

For the second and third parts, ${ }^{45} 4632$ patients meeting ACR criteria for knee OA were recruited from outpatient clinics. The mean age of the patients was 63.1 years, $74 \%$ were female, and the average body mass index was 31 . Grade IV radiographs 
were an exclusion criterion. MRI acquisition was performed at baseline and 6, 12, 18, and 24 months of follow up. For the second study, ${ }^{45}$ the images were randomised and the cartilage volumes of the total cartilage, as well as the medial and lateral compartments, were analysed. The variables included the Western Ontario and McMaster Questionnaire (WOMAC), visual analogue scales of pain, global evaluation (patient and doctor) of the disease, consumption of other drugs, and physical examination of the knee. The data on knee OA progression (cartilage volume losses in percentage from baseline) computed at all the time points of follow up showed as early as six months a striking and significant loss of cartilage. ${ }^{46} \mathrm{~A}$ higher loss was seen in the medial compartment. Interestingly, two populations described as "fast" and "slow" progressors were identified. These data support the strong advantage of quantitative MRI over existing techniques in that MRI is able to detect significant changes in knee OA cartilage volume as early as six months.

In the third study, ${ }^{46}$ the severity of OA changes in the medial and lateral menisci was evaluated using a semiquantitative scale for degeneration, tear, and extrusion. A significant association was found between meniscal tear and extrusion at baseline but not meniscal degeneration. However, no significant change was seen in the three parameter scores over the two year observation time. When the meniscal alterations were compared with the loss of cartilage volume over time, a significant difference in global cartilage volume loss was observed between severe medial meniscal tear and no tear. There was an even greater difference between the medial meniscal changes in the medial compartment and the cartilage volume loss at the medial compartment. Similarly, a highly significant difference was found between the presence of a medial meniscal extrusion and the loss of cartilage volume at the medial compartment. A multilinear regression analysis showed that medial meniscal extrusion of the anterior horn was predictive of the global cartilage volume loss of the medial or lateral compartment. These data show that meniscal tear and extrusion are key parameters which must be addressed when predicting the progression of knee OA.

\section{CONCLUSION}

This short review has highlighted the most recent advances in the understanding of the pathophysiology of OA. Several interesting new approaches to the treatment of this disease are now being explored. New classes of molecules that inhibit one or more of the disease processes are under evaluation.

The progression of cartilage degradation in knee OA is a key parameter that needs to be quantified in any study of the efficacy of therapeutic interventions for this disease. The potential of the MRI approach is obvious, as it provides a clear advantage over any of the other existing technologies. Moreover, our MRI system makes a highly reliable quantification of cartilage volume possible. Such technology will be critical for the analysis of disease progression over time and should reduce the number of patients needed in clinical trials, improve the retention of these patients, and reduce the overall costs and the length of clinical trials.

\section{Authors' affiliations}

J-P Pelletier, J Martel-Pelletier, University of Montreal Hospital Center, Notre-Dame Hospital, Osteoarthritis Research Unit, Montreal, Quebec,

Canada

Correspondence to: Professor J-P Pelletier, University of Montreal Hospital Center, Notre-Dame Hospital, Osteoarthritis Research Unit, 1560 rue Sherbrooke Est, Montreal, Quebec, H2L 4M1 Canada; dr@ippelletier.ca

\section{REFERENCES}

1 Pelletier JP, Martel-Pelletier J, Howell DS. Etiopathogenesis of osteoarthritis. In: Koopman WJ, ed. Arthritis \& allied conditions. A textbook of rheumatology. Baltimore: Williams \& Wilkins, 2001:2195-245

2 Towheed TE, Hochberg MC. A systematic review of randomized controlled trials of pharmacological therapy in osteoarthritis of the knee, with an emphasis on trial methodology. Semin Arthritis Rheum 1997:26:755-70.

3 American College of Rheumatology Subcommittee on Osteoarthritis Guideline. Recommendations for the medical management of osteoarthritis of the hip and knee: 2000 update. Arthritis Rheum 2000;43:1905-15.

4 Creamer $\mathbf{P}$. Intra-articular corticosteroid treatment in osteoarthritis. Curr Opin Rheumatol 1999;11:417-21.

5 McCarthy GM. Role of crystal deposition in the osteoarthritic joint. In: Reginster JY, Pelletier JP, Martel-Pelletier J, Henrotin Y, eds. Osteoarthritis: clinical and experimental aspects. Berlin: Springer-Verlag, 1999:210-27.

6 Raynauld JP, Buckland-Wright C, Ward R, Choquette D, Haraoui B, Martel-Pelletier J, et al. Safety and efficacy of long-term intraarticular steroid injections in osteoarthritis of the knee: a randomized, double-blind, placebo-controlled trial. Arthritis Rheum 2003;48:370-7.

7 Pelletier JP, Martel-Pelletier J, Abramson SB. Osteoarthritis, an inflammatory disease: potential implication for the selection of new therapeutic targets. Arthritis Rheum 2001;44:1237-47.

8 Caron JP, Fernandes JC, Martel-Pelletier J, Tardif G, Mineau F, Geng C et al. Chondroprotective effect of intraarticular injections of interleukin-1 receptor antagonist in experimental osteoarthritis: suppression of collagenase-1 expression. Arthritis Rheum 1996;39:1535-44

9 Fernandes JC, Tardif G, Martel-Pelletier J, Lascau-Coman V, Dupuis M, Moldovan F, et al. In vivo transfer of interleukin-1 receptor antagonist gene in osteoarthritic rabbit knee joints: prevention of osteoarthritis progression. Am J Pathol 1999; 154:1159-69.

10 Pelletier JP, Caron JP, Evans CH, Robbins PD, Georgescu HI, Jovanovic $\mathrm{D}$, et al. In vivo suppression of early experimental osteoarthritis by IL-Ra using gene therapy. Arthritis Rheum 1997;40:1012-19.

11 Saha N, Moldovan F, Tardif G, Pelletier JP, Cloutier JM, Martel-Pelletier J. Interleukin-1 $\beta$-converting enzyme/Caspase-1 in human osteoarthritic tissues: localization and role in the maturation of IL-1 $\beta$ and IL-18. Arthritis Rheum 1999;42:1577-87

12 Martel-Pelletier J, Tardif G, Fernandes JC, Pelletier JP. Metalloproteases and their modulation as treatment in osteoarthritis. In: Tsokos GC, ed. Molecular rheumatology. Totowa, NJ: Humana Press Inc, 2000:499-514.

13 Pelletier JP, Fernandes JC, Jovanovic DV, Reboul P, Martel-Pelletier J. Chondrocyte death in experimental osteoarthritis is mediated by MEK $1 / 2$ and p38 pathways: role of COX-2 and iNOS. J. Rheumatol $2001 ; 28: 2509-19$

14 Pelletier JP, Jovanovic DV, Lascau-Coman V, Fernandes JC, Manning PT, Connor JR, et al. Selective inhibition of inducible nitric oxide synthase reduces progression of experimental osteoarthritis in vivo: possible link with the reduction in chondrocyte apoptosis and caspase 3 level. Arthritis Rheum 2000:43:1290-9.

15 Boileau C, Martel-Pelletier J, Moldovan F, Jouzeau JY, Netter P, Manning PT, et al. The in situ up-regulation of chondrocyte interleukin-1-converting enzyme and interleukin- 18 levels in experimental osteoarthritis is mediated by nitric oxide. Arthritis Rheum 2002;46:2637-47.

16 Raynauld JP. Magnetic resonance imaging of articular cartilage: toward a redefinition of "primary" knee osteoarthritis and its progression. J Rheumatol 2002;29:1809-10.

17 Peterfy CG. Imaging of the disease process. Curr Opin Rheumato 2002; 14:590-6.

18 Eckstein F, Stammberger T, Priebsch J, Englmeier KH, Reiser M. Effect of gradient and section orientation on quantitative analysis of knee joint cartilage. J Magn Reson Imaging 2000;1 1:469-70.

19 Frank LR, Wong EC, Luh WM, Ahn JM, Resnick D. Articular cartilage in the knee: mapping of the physiologic parameters at MR imaging with a local gradient coil: preliminary results. Radiology 1999;210:241-6.

20 Haubner M, Eckstein F, Schnier M, Losch A, Sittek H, Becker C, et al. A non-invasive technique for 3-dimensional assessment of articular cartilage thickness based on MRI. Part 2. Validation using CT arthrography. Magn Reson Imaging 1997; 15:805-13.

21 Hohe J, Faber S, Stammberger T, Reiser M, Englmeier KH, Eckstein F. A technique for $3 \mathrm{D}$ in vivo quantification of proton density and magnetization transfer coefficients of knee joint cartilage. Osteoarthritis Cartilage 2000;8:426-33

22 Peterfy CG, Genant HK. Emerging applications of magnetic resonance imaging in the evaluation of articular cartilage. Radiol Clin North Am 1996;34:195-213, ix.

23 Eckstein F, Winzheimer $M$, Westhoff J, Schnier M, Haubner $M$, Englmeier $\mathrm{KH}$, et al. Quantitative relationships of normal cartilage volumes of the human knee joint: assessment by magnetic resonance volumes of the human knee joint: assessment by magr
imaging. Anat Embryol (Berl) 1998;197:383-90.

24 Kshirsagar AA, Watson PJ, Tyler JA, Hall LD. Measurement of localized cartilage volume and thickness of human knee joints by computer analysis of three-dimensional magnetic resonance images. Invest Radiol 1998;33:289-99

25 Stammberger T, Herberhold C, Faber S, Englmeier KH, Reiser $M$, Eckstein F. A method for quantifying time dependent changes in MR signal intensity of articular cartilage as a function of tissue deformation in intact joints. Med Eng Phys 1998;20:741-9

26 Calvo E, Palacios I, Delgado E, Ruiz-Cabello J, Hernandez P, Sanchez-Pernaute O, et al. High-resolution MRI detects cartilage swelling 
at the early stages of experimental osteoarthritis. Osteoarthritis Cartilage 2001:9:463-72.

27 McNicholas MJ, Brooksbank AJ, Walker CM. Observer agreement analysis of MRI grading of knee osteoarthritis. J R Coll Surg Edinb 1999:44:31-3.

28 Mosher TJ, Dardzinski BJ, Smith MB. Human articular cartilage: influence of aging and early symptomatic degeneration on the spatial variation of T2: preliminary findings at $3 \mathrm{~T}$. Radiology 2000;21 4:259-66.

29 Bredella MA, Tirman PF, Peterfy CG, Zarlingo M, Feller JF, Bost FW, et al. Accuracy of T2-weighted fast spin-echo MR imaging with fat saturation in detecting cartilage defects in the knee: comparison with arthroscopy in 130 patients. AJR Am J Roentgenol 1999;172:1073-80.

30 Drape JL, Pessis E, Auleley GR, Chevrot A, Dougados M, Ayral X. Quantitative MR imaging evaluation of chondropathy in osteoarthritic knees. Radiology 1998;208:49-55.

31 Kladny B, Bail H, Swoboda B, Schiwy-Bochat H, Beyer WF, Weseloh G. Cartilage thickness measurement in magnetic resonance imaging. Osteoarthritis Cartilage 1996:4:181-6.

32 Uhl M, Ihling C, Allmann KH, Laubenberger J, Taver U, Adler $\mathrm{CP}$, et al. Human articular cartilage: in vitro correlation of MRI and histologic findings. Eur Radiol 1998;8:1123-9.

33 Cohen ZA, McCarthy DM, Kwak SD, Legrand P, Fogarasi F, Ciaccio EJ et al. Knee cartilage topography, thickness, and contact areas from MRI in-vitro calibration and in-vivo measurements. Osteoarthritis Cartilage 1999;7:95-109.

34 Pilch L, Stewart C, Gordon D, Inman R, Parsons K, Pataki I, et al. Assessment of cartilage volume in the femorotibial joint with magnetic resonance imaging and 3D computer reconstruction. J Rheumatol 1994:21:2307-21.

35 Piplani MA, Disler DG, McCauley TR, Holmes TJ, Cousins JP. Articular cartilage volume in the knee: semiautomated determination from three-dimensional reformations of MR images. Radiology 1996; 198:855-9.

36 Eckstein F, Schnier M, Haubner M, Priebsch J, Glaser C, Englmeier KH et al. Accuracy of cartilage volume and thickness measurements with magnetic resonance imaging. Clin Orthop 1998;352:137-48.
37 Peterfy CG, van Dijke CF, Janzen DL, Gluer CC, Namba R, Majumdar $\mathrm{S}$, et al. Quantification of articular cartilage in the knee with pulsed saturation transfer subtraction and fat-suppressed MR imaging: optimization and validation. Radiology 1994;192:485-91.

38 Stammberger T, Eckstein F, Englmeier KH, Reiser M. Determination of 3D cartilage thickness data from MR imaging: computational method and reproducibility in the living. Magn Reson Med 1999;41:529-36.

39 Cicuttini F, Forbes A, Asbeutah A, Morris K, Stuckey S. Comparison and reproducibility of fast and conventional spoiled gradient-echo magnetic resonance sequences in the determination of knee cartilage volume. J Orthop Res 2000; 18:580-4

40 Burgkart R, Glaser C, Hyhlik-Durr A, Englmeier KH, Reiser M, Eckstein $F$. Magnetic resonance imaging-based assessment of cartilage loss in severe osteoarthritis: accuracy, precision, and diagnostic value. Arthritis Rheum 2001;44:2072-7.

41 Wluka AE, Stuckey S, Snaddon J, Cicuttini FM. The determinants of change in tibial cartilage volume in osteoarthritic knees. Arthritis Rheum 2002;46:2065-72.

42 Cicuttini FM, Wluka AE, Stuckey SL. Tibial and femoral cartilage changes in knee osteoarthritis. Ann Rheum Dis 2001;60:977-80.

43 Kauffmann C, Gravel P, Godbout B, Gravel A, Beaudoin G, Raynauld $\mathrm{J}-\mathrm{P}$, et al. Computer-aided method for quantification of cartilage thickness and volume changes using MRI: validation study using a synthetic model. IEEE Trans Biomed Eng 2003:in press.

44 Raynauld JP, Kaufmann C, Beaudoin G, Berthiaume M, de Guise JA, Bloch DA, et al. Reliability of a quantification imaging system using magnetic resonance images to measure cartilage thickness and volume in human normal and osteoarthritic knees. Osteoarthritis Cartilage 2003;11:351-60.

45 Raynauld JP, Pelletier JP, Beaudoin G, Berthiaume M, de Guise JA, Bloch DA, et al. A two-year study in osteoarthritis patients following the progression of the disease by magnetic resonance imaging using a nove quantification imaging system [abstract]. Arthritis Rheum 2002;46:S150.

46 Raynaud J-P, Pelletier J-P, Beaudoin G, Berthiaume M-J, de Guise J, Bloch DA, et al. Meniscal damage is strongly associated with the progression of knee osteoarthritis as assessed by a quantification imaging system using magnetic resonance imaging [abstract]. Ann Rheum Dis 2003;62:SAT0145. 